\title{
Evaporation of liquid nitrogen droplets in superheated immiscible liquids
}

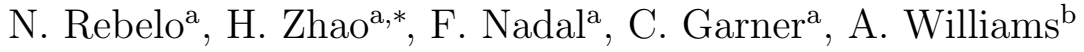 \\ ${ }^{a}$ Wolfson School of Mechanical, Electrical and Manufacturing Engineering, Loughborough \\ University,Loughborough, Leicestershire, LE11 3TU, UK \\ ${ }^{b}$ Department of Mechanical Engineering, University of Chester, Chester, CH1 4BJ, UK
}

\begin{abstract}
Liquid nitrogen or other cryogenic liquids have the potential to replace or augment current energy sources in cooling and power applications. This can be done by the rapid evaporation and expansion processes that occur when liquid nitrogen is injected into hotter fluids in mechanical expander systems. In this study, the evaporation process of single liquid nitrogen droplets when submerged into n-propanol, methanol, n-hexane, and n-pentane maintained at $294 \mathrm{~K}$ has been investigated experimentally and numerically. The evaporation process is quantified by tracking the growth rate of the resulting nitrogen vapour bubble that has an interface with the bulk liquid. The experimental data suggest that the bubble volume growth is proportional to the time and the bubble growth rate is mainly determined by the initial droplet size. A comparison between the four different bulk liquids indicates that the evaporation rate in n-pentane is the highest, possibly due to its low surface tension. A scaling law based on the pure diffusion-controlled evaporation of droplet in open air environment has been successfully implemented to scale the experimental data. The deviation between the scaling law predictions and the experimental data for 2-propanol, methanol and nhexane vary between 4-30\% and the deviation for n-pentane was between $24-65 \%$. The more detailed bubble growth rates have been modelled by a heuristic one-dimensional, spherically symmetric quasi-steady-state confined model, which can predict the growth trend well but consistently underestimate the growth rate. A fixed effective thermal conductivity is then introduced to account for the complex dynamics of the droplet inside the bubble and the subsequent convective processes in the surrounding vapour, which leads to a satisfactory quantitative prediction of the growth rate.
\end{abstract}

Keywords: Liquid nitrogen; Cryogenic droplets; Evaporation; superheated immiscible liquid

\section{Introduction}

The past four decades have seen a rise of about $90 \%$ in global $\mathrm{CO}_{2}$ emission rates, with those from fossil fuel combustion and industrial processes accounting for $78 \%$ of this [1]. To mitigate the negative impact of $\mathrm{CO}_{2}$ emis- sions on the environment, cleaner energy systems need to be developed. One of the potential energy systems with zero tailpipe $\mathrm{CO}_{2}$ emissions is utilising the evaporation process of cryogens, such as Liquid Nitrogen $\left(\mathrm{LN}_{2}\right)$ in a constrained volume. It is important to 
note that unlike petrol and diesel, $\mathrm{LN}_{2}$ does not contain any useful chemical energy but is rather a carrier of thermal energy. Over the last few decades, two main methods of utilizing the cold energy of cryogenic liquids have been investigated. The first method consists of evaporating the cryogenic liquid under pressure via indirect contact exchange with an external heat source and then expanding the pressurized gas in an expander to harvest expansion work, as done in the case of the CooLN2NCAR [2] and the Dearman engine [3]. These systems typically need a large surface area heat exchanger to achieve the high evaporation rate, which can make the system costly and bulky. In addition, some of the pressure and hence the mechanical work will be lost during the gas intake process (i.e. the pumping loss). The second method is to inject the cryogen liquid directly into a hot fluid (gas or liquid) inside a piston engine cylinder to produce a high pressure gas through the fast evaporation of the cryogen when the engine is near its smallest volume (i.e. when the piston is near the top dead centre). This high pressure pushes the piston to produce useful work. This approach could avoid the pumping loss through the gas intake process. One such system was investigated by Clarke [4]. The tests were performed on a prototype cryogenic engine. Nitrogen with a normal boiling point at $77 \mathrm{~K}\left(-196{ }^{\circ} \mathrm{C}\right)$ was expanded by heat exchange with air or water allowing useful work to be extracted. Upon direct injection of $\mathrm{LN}_{2}$ into the water in the cylinder, a specific work of only $12.8 \mathrm{~kJ} \mathrm{~kg}^{-1}$ was obtained,while the engine seized after a few cycles due to the freezing of engine components at low temperature. Clarke attributed this poor performance to the incomplete vaporization of $\mathrm{LN}_{2}$. An indirect heat exchange system (nitrogen vaporized and pressurized to 13.3 bar externally) with the same en- gine, delivered a maximum specific work of $128 \mathrm{~kJ} \mathrm{~kg}^{-1}$ which was still low compared to the exergy $\left(744 \mathrm{~kJ} \mathrm{~kg}^{-1}\right.$ at NTP (i.e. 1 bar pressure at $293 \mathrm{~K}$ ) of $\mathrm{LN}_{2}$. Clarke found that for the indirect approach, a large exergy loss took place across the cryogenic injector $\left(\sim 350 \mathrm{~kJ} \mathrm{~kg}^{-1}\right)$ that was designed for vaporization and pre-pressurization of $\mathrm{LN}_{2}$ in an isochoric process followed by injection of pressurised gaseous nitrogen into the engine cylinder. Therefore, a direct injecting approach with a significantly improved evaporation rate could potentially be the more efficient system. While evaporation of cryogens through indirect heat transfer has been well studied, there is a paucity of published research available on the evaporation of cryogens immersed in another fluid, especially liquid.The evaporation rate of freely falling $\mathrm{LN}_{2}$ droplets in air was investigated by Awonorin [5]in 1989. By employing high-speed photography to estimate the droplet size and velocity with time, he calculated the average heat transfer coefficient from the slopes of the droplet diameter versus time curve on an average basis. He also provided an empirical heat transfer correlation in terms of Nusselt number, $\mathrm{Nu}$, and obtained higher Nusselt numbers than other theoretical predictions. An effort to quantify indirectly the heat transfer during the injection of an $\mathrm{LN}_{2}$ jet into a pool of water was made in the year 2006 by Wen et al. [6]. The average pressure increase rate obtained by them was $265.6 \mathrm{kPa}$ $\mathrm{s}^{-1}$. A thermodynamic analysis based on the measured pressure trace and the assumptions of a quasi-equilibrium system and the ideal gas law suggested that the heat transfer coefficients vary between $4-25 \mathrm{~kW} \mathrm{~m}^{2} \mathrm{~K}^{-1}$. A major uncertainty in this calculation was from the estimation of the interfacial area between nitrogen and water. In 2010, Clarke et al. [7] performed a similar experiment as Wen et 
al. albeit with a smaller free volume $(50 \mathrm{ml})$ and higher cryogenic liquid injection pressures. Their results gave much higher pressure rise rates of approximately $19000 \mathrm{kPa}$ $\mathrm{s}^{-1}$. They observed that the maximum pressurisation occurred when the $\mathrm{LN}_{2}$ jet collided with the bottom wall of the chamber. By using the measured pressure trace, Clarke et al. [7] calculated the total heat transfer to the injected nitrogen and found it to be $224 \mathrm{~J}$. The heat transfer to nitrogen from the vessel walls was estimated to be $\sim 32 \mathrm{~J}$ based on an empirical correlation and an estimated interfacial area. Another experimental work on $\mathrm{LN}_{2}$ jet boiling in water was conducted in 2014 by Nakoryakov et al. [8]. This work was aimed at developing a novel shock-wave method for gas hydrate formation. Capsules filled with $\mathrm{LN}_{2}$ were pressurized with helium gas until they ruptured and injected $\mathrm{LN}_{2}$ into the water. They observed a liquid core surrounded by vapour. They used a similar injection pressure as Clarke et al. [7] with a larger free volume $(67.4$ - $269.6 \mathrm{ml})$ but obtained pressure rise rates that were an order of magnitude smaller $\left(3611 \mathrm{kPa} \mathrm{s}^{-1}\right)$.

In summary, although earlier works have reported some information on heat transfer rates based on the measured pressure rise rates, the large uncertainty in the interfacial heat transfer area casts doubt on the accuracy of the effective heat transfer rate. In addition, instead of a liquid cryogen jet, a well atomised cryogenic liquid could be used to achieve a higher evaporation rate due to the signficiantly increased interfacial area. Considering that high evaporation rates are crucial for power generation in cryogenic energy systems, a more accurate quantification of the evaporation rate of a cryogenic spray is required to analyse and optimise system designs. Since a cryogenic spray usually consists of numerous cryogenic droplets, the log- ical first step towards the full spray study is to measure the heat transfer rate of a single $\mathrm{LN}_{2}$ droplet while boiling in a hotter bulk liquid. In addition, a simple conductive model based on one-dimensional (spherical coordinate) Quasi-Steady State Approximations (QSSA) has been proposed to predict the time-profile of the bubble radius and the rate of heat transfer during evaporation. In this paper, the experimental set up, materials and method are detailed in Section 2. The experimental results are analysed and discussed in Section 3. The semi-analytical model is compared to experimental data in Section 4. Conclusions from this work are presented in Section 5 .

\section{Experimental setup and measure- ment methods}

In this section, the experimental setup used for visualising the $\mathrm{LN}_{2}$ droplet evaporation process and quantifying the heat transfer rate will be discussed. The main hardware included an $\mathrm{LN}_{2}$ injector, a test section and an optical imaging system. $\mathrm{A} \mathrm{LN}_{2}$ droplet having a density $\rho_{d}=807 \mathrm{~kg} \mathrm{~m}^{-3}$, viscosity $\mu_{d}=161 \times 10^{-3} \mathrm{~Pa}$, surface tension $\sigma_{d}=11$ $\mathrm{mN} \mathrm{m}^{-1}$ ) was injected into different bulk liquids (properties are defined in Table 2). The evaporation process was recorded by the imaging system for post-processing to quantify the evaporation rate.

\section{1. $L N_{2}$ drop injector and test cell}

A vacuum insulated $\mathrm{LN}_{2}$ injector, as shown in Fig.1, was built in-house to generate cryogenic droplets in a controlled manner. A cryogenic solenoid valve (BCryo, Gems Sensors \& Controls) which had an opening time of $50 \mathrm{~ms}$ was used to control the droplet size and the generation rate. Downstream of the valve, a luer lock fitting was used to connect a stainless steel needle. 


\section{Nomenclature}

\begin{tabular}{|c|c|c|}
\hline$A$ & surface area $\left(\mathrm{mm}^{2}\right)$ & Greek symbols \\
\hline$a$ & capillary length (mm) & diffusion parameter $\left(\mathrm{m}^{2} \mathrm{~s}^{-1}\right)$ \\
\hline Bo & Bond number (-) & thermal diffusivity $\left(\mathrm{m}^{2} \mathrm{~s}^{-1}\right)$ \\
\hline$c_{p}$ & specific heat $\left(\mathrm{J} \mathrm{kg}^{-1} \mathrm{~K}^{-1}\right)$ & density $\left(\mathrm{kg} \mathrm{m}^{-3}\right)$ \\
\hline$D$ & diameter $(\mathrm{mm})$ & dynamic viscosity $\left(\mathrm{Pa} \mathrm{s}^{-1}\right)$ \\
\hline$g$ & gravitational acceleration $\left(\mathrm{m} \mathrm{s}^{-2}\right)$ & kinematic viscosity $\left(\mathrm{m}^{2} \mathrm{~s}^{-1}\right)$ \\
\hline$h_{f g}$ & latent heat of vaporisation $\left(\mathrm{J} \mathrm{kg}^{-1}\right)$ & surface tension $\left(\mathrm{mN} \mathrm{m}^{-1}\right)$ \\
\hline$k$ & thermal conductivity $\left(\mathrm{W} \mathrm{m}^{-1} \mathrm{~K}^{-1}\right)$ & vapour layer thickness (mm) \\
\hline $\mathcal{M}_{N_{2}}$ & molar mass $\left(\mathrm{g} \mathrm{mol}^{-1}\right)$ & dimensionless time \\
\hline$m_{d}$ & droplet mass (kg) & scaling coefficient $(-)$ \\
\hline$n$ & number of moles (mol) & corrected scaling coefficient (-) \\
\hline$N T P$ & normal temperature pressure & \\
\hline$P$ & pressure $(\mathrm{Pa})$ & Subscripts \\
\hline$q$ & heat flux $\left(\mathrm{W} \mathrm{cm}^{-2}\right)$ & bubble \\
\hline$r$ & droplet radius $(\mathrm{mm})$ & droplet \\
\hline$R$ & bubble radius (mm) & bulk liquid \\
\hline $\mathcal{R}$ & ideal gas constant $\left(\mathrm{J} \mathrm{mol}^{-1} \mathrm{~K}^{-1}\right)$ & initial \\
\hline$\dot{R}$ & radial velocity $\left(\mathrm{cm} \mathrm{s}^{-1}\right)$ & atmosphere \\
\hline$R e$ & Reynolds number (-) $\left(\mathrm{cm} \mathrm{s}^{-1}\right)$ & effective \\
\hline$T$ & temperature $(\mathrm{K})$ & inertial \\
\hline $\bar{T}$ & mean temperature $(\mathrm{K})$ & viscous \\
\hline$t$ & time (ms) & surface tension \\
\hline$t_{e}$ & evaporation time (ms) & \\
\hline$v$ & droplet volume $\left(\mathrm{mm}^{3}\right)$ & \\
\hline$V$ & bubble volume $\left(\mathrm{mm}^{3}\right)$ & \\
\hline
\end{tabular}

The injector was operated at atmospheric pressure in the current work. The outer vacuum housing was evacuated to 8 mbar using a diaphragm vacuum pump (Leybold). An electrical feedthrough was employed to connect the lead wires of the solenoid valve without breaking the vacuum.

The test cell was an optical glass chamber (Hellma) with inner dimensions of $50 \times 50 \times 50$ $\mathrm{mm}$. The sides of this chamber had a parallelism of $20 \mu \mathrm{m}$. The centre point of the glass chamber was carefully aligned with the injec- tor. An acrylic cover with a central $4 \mathrm{~mm}$ diameter hole was placed on the glass chamber so that only those droplets in the plane of focus could enter the chamber.

\subsection{Optical Imaging technique}

Two identical backlight imaging systems, as shown in Fig.2, were employed at orthogonal directions to visualise the droplet evaporation process. Two Multi-LED light sources (GSVITEC), each having a power of 7700 lumens were used for the illumination of each view. A condenser lens was used in front of the light source to increase the amount 


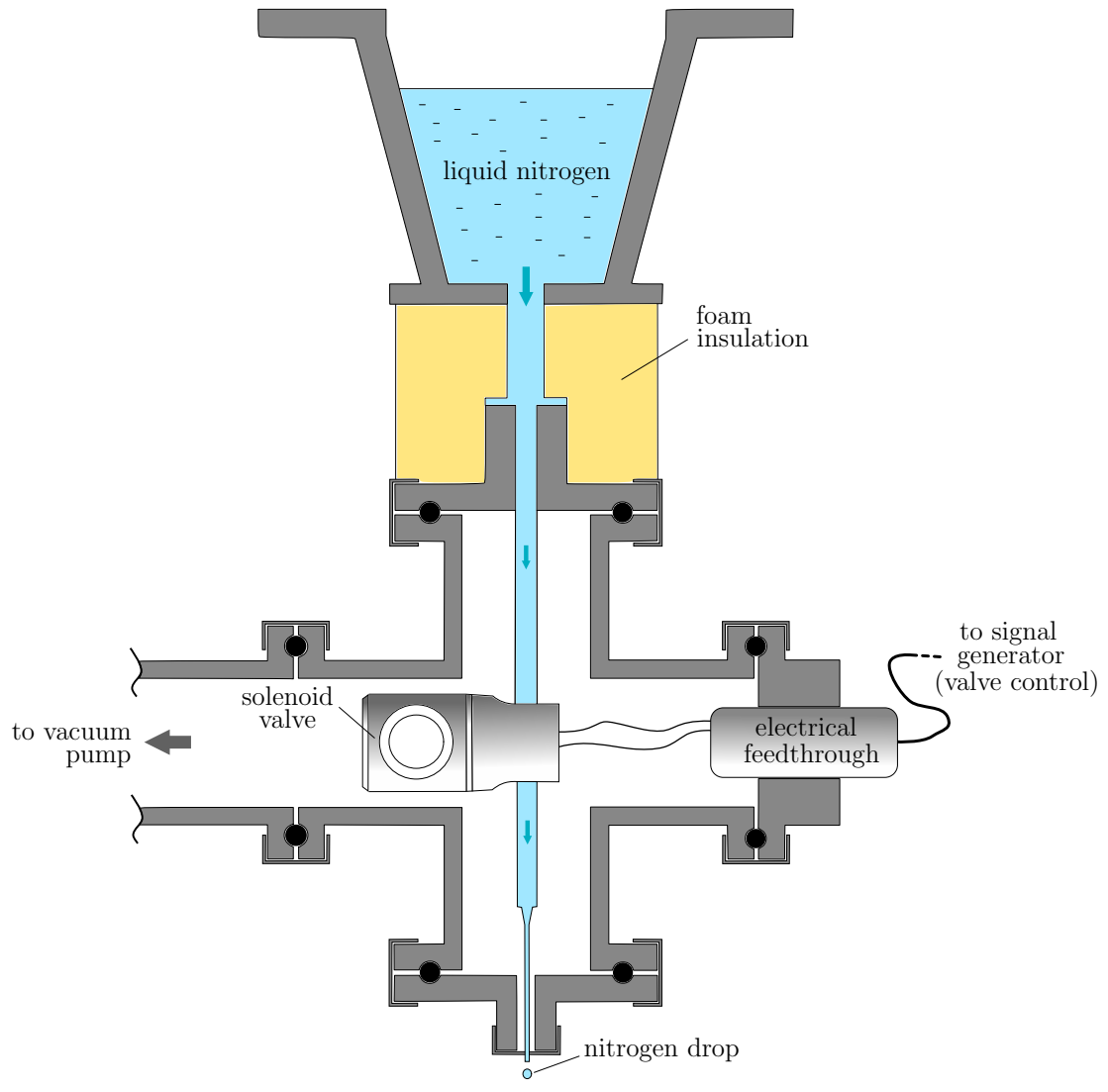

Figure 1: Droplet generation system. The needle tip was located 400 millimeters above the liquid/air inteface.

of light incident on the object being imaged. A ground glass diffuser was placed after this lens to provide a more uniform background. An iris of $25 \mathrm{~mm}$ in diameter was placed in front of the diffuser to ensure that light passed only through the desired field of view (FoV) without any undesirable reflections. An aspheric condenser lens (diam. $50.8 \mathrm{~mm}$ ) was used to provide partial collimation of the light beam for the detection of fine changes in refractive index. This optical system was preferred to a parallel light shadowgraphy system because (i) the amount of light required to visualise the highly mobile $\mathrm{LN}_{2}$ droplet and bubble was too challenging for a point LED source with collimation, as required by the shadowgraphy technique, (ii) a collimated shadowgraphy system would not have allowed a proper visualization of the nitrogen droplet within the bubble.

Two high speed cameras (VEO 710L, Phantom) set up at a resolution of $1200 \times 800$ pixels, and a frame rate of $2000 \mathrm{fps}$ were used to capture the images from two orthogonal views to compute the volume and surface area of the interface. In principle, a perfect analytical reconstruction of the 3D interface needs three views. However, in our experiments, the shapes of the bubbles having diameter $\left(D_{b}\right) \leq 3.4 \mathrm{~mm}$ while growing at the rates $(\dot{R}) \leq 1.8 \mathrm{~cm} \mathrm{~s}^{-1}$ in an infinite incompressible liquid (i.e. Bond number $B o=g \Delta \rho D_{b}^{2} / \sigma_{l} \leq 4$ and a Reynolds number $R e=\rho_{b} \dot{R} D_{b} / \mu_{b} \leq 9$, where $g=$ 


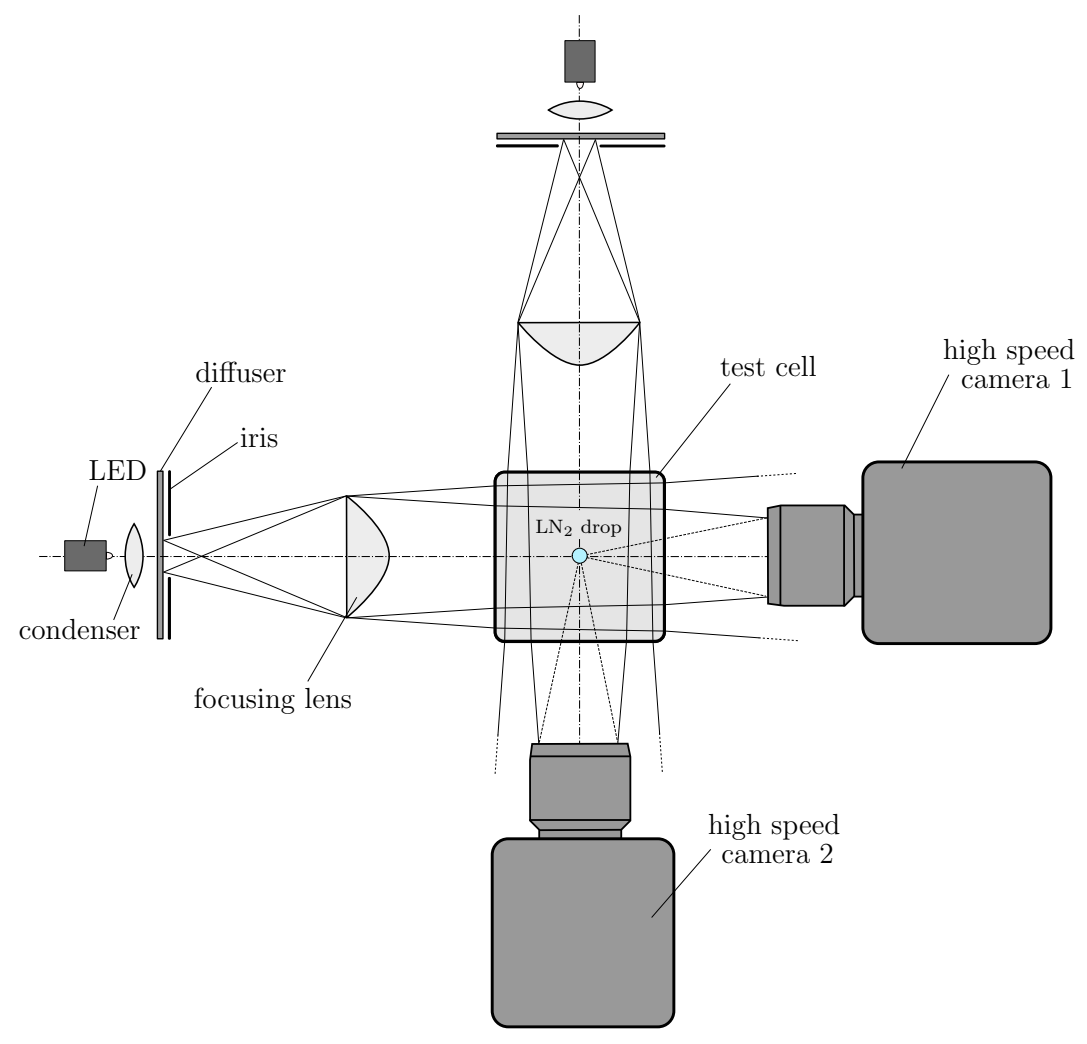

Figure 2: Optical setup. The enlightment system is not collimated to enable the visualization of the nitrogen droplet within the bubble.

$9.81 \mathrm{~m} \mathrm{~s}^{-2}$ is the gravitational acceleration, Two $60 \mathrm{~mm}$ focal length macro lenses (Nikon) $\Delta \rho=\left|\rho_{b}-\rho_{l}\right|$ is the absolute value of den- along with $20 \mathrm{~mm}$ extension rings were used sity difference between the bubble and the bulk liquid, $\rho_{b}=1.8 \mathrm{~kg} \mathrm{~m}^{-3}, \rho_{l}=780.86 \mathrm{~kg}$ $\mathrm{m}^{-3}$ are the densities of the nitrogen bubble and the bulk liquid (2-propanol) respectively, $\sigma_{l}=21.74 \mathrm{mN} \mathrm{m}^{-1}$ is the surface tension of the bulk liquid interfaced with the bubble, $\mu_{b}=12.2 \times 10^{-6} \mathrm{~Pa} \mathrm{~s}$ is the viscosity of the bubble) are ellipsoidal with an aspect ratio close to unity, as shown by Clift et al. [9]. Therefore, the error in estimated volume caused by the absence of the third plane was small and hence not considered. In addition, the near spherical bright areas found in both views of bubbles recorded indicated that the bubble shape was indeed close to spherical or ellipsoidal with an aspect ratio close to unity. to achieve $\times 1.42$ magnification. An external signal generator was used to synchronize both cameras and the solenoid valve inside the injector.

\subsection{Calibration and Data Analysis}

Prior to commencing experiments, the imaging system was precisely focussed using a $100 \mu \mathrm{m}$ spaced glass grid (Thorlabs). The FoV and magnification of the optical system were calibrated using a $500 \mu \mathrm{m}$ spaced glass grid. The calibration shows the pixel size is $14.0845 \mu \mathrm{m} /$ pixel and the field of view is $16.8 \times 11.27 \mathrm{~mm}$. The depth of field (DoF) of the imaging system was computed using a grid of $250 \mu \mathrm{m}$ dot spacing which estimated 


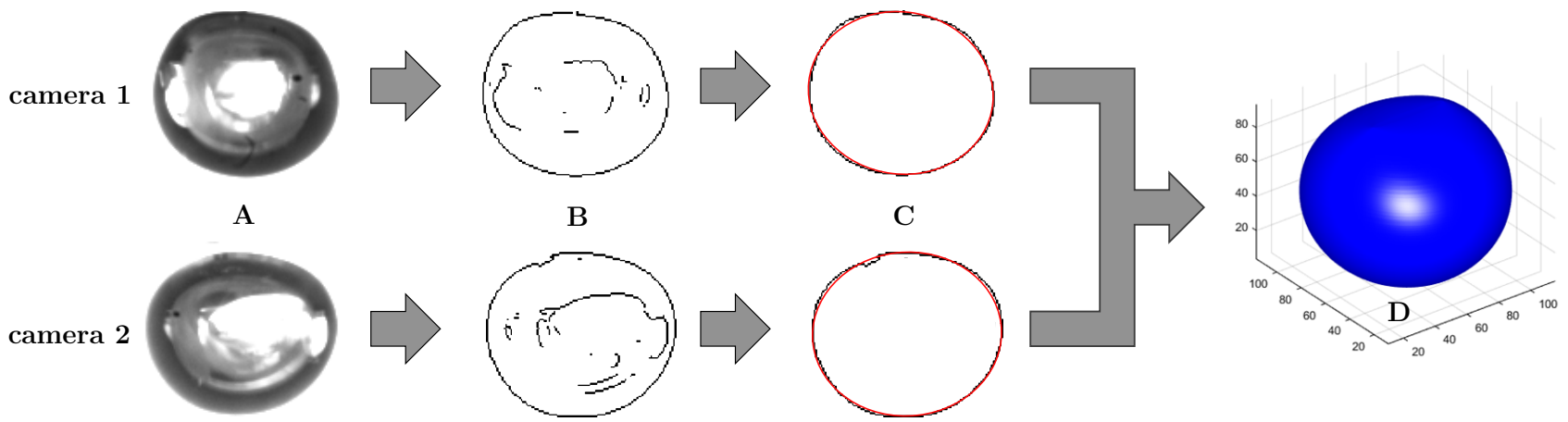

Figure 3: Volume computation method. Two synchronized images provided by the two cameras (A) are used to perform an edge detection (B) that enables a fitting by an ellipse (red curve in C). Note that the 3D reconstructed shape (D) provides the correct volume (within the measurement uncertainty), but the shape may differ from the actual shape due to the absence of a third view to define the exact orientation of the droplet.

the depth of field to be approximately $5 \mathrm{~mm}$. The calibration images were then analysed for distortion error using an open source image processing software Image J, which revealed negligible distortion. The captured images were then post-processed and analysed using a Matlab programme, as shown in Fig.3. A programme built in Matlab processed each pair of images from the two cameras using either the Sobel or Canny edge detection functions depending on the image contrast, taking advantage of these techniques as proposed by Burger \& Burge [10] to binarise the images. An ellipse closest to the shape the nitrogen droplet/vapour bubble was then estimated using the randomized Hough transform [11]. A 3D point cloud was finally generated from the two views, with a volume computation using the alpha shape function in Matlab.

The volume computation algorithm was validated using a spherical ceramic ball of grade 3 precision $( \pm 0.8 \mu \mathrm{m})$. The mean diameter of the ball was confirmed to be 3.169 $\mathrm{mm}$ using a micrometre gauge. Other symmetric objects built to scale using NX soft- ware (Siemens) were also used for the validation of the algorithm. Table 1 below shows that the error in computed volumes and surface areas for such smooth symmetric geometries is small. The main source of these computed errors is from the assumption that the droplets/bubbles have a regular smooth surface, which are consistent with the recorded images of the droplets and bubbles. As the refractive index change between $\mathrm{LN}_{2}(\simeq 1.2)$ and its vapour $(\simeq 1)$ is small, the program needed to be validated for a poorly contrasted boundary. As in the earlier validation, the same ceramic ball was captured at different contrasts controlled by the exposure time. In the worst case, this led to a $\pm 3.86 \%$ error in the ball diameter, $\pm 5.45 \%$ in the surface area, and $\pm 6.68 \%$ in the ball volume.

\section{Experimental results and discussion}

\subsection{Qualitative results from high-speed visu- alization}

The process begins with the break off of a $\mathrm{LN}_{2}$ jet from the injector due to hydrodynamic instabilities, such as the Rayleigh- 
Table 1: Computed volumes $\left(\mathrm{mm}^{3}\right)$ and surface areas $\left(\mathrm{mm}^{2}\right)$ obtained using the Matlab programme (note - $a$ : measured value; $b$ : geometry built to scale in Siemens NX 10.0 software).

\begin{tabular}{ccccccc}
\hline Object & $\begin{array}{c}\text { Actual } \\
\text { Volume }\end{array}$ & $\begin{array}{c}\text { Computed } \\
\text { Volume }\end{array}$ & $\begin{array}{c}\text { Volume } \\
\text { error (\%) }\end{array}$ & $\begin{array}{c}\text { Actual Surf. } \\
\text { Area }\end{array}$ & $\begin{array}{c}\text { Computed } \\
\text { Surf. Area }\end{array}$ & $\begin{array}{c}\text { Surface Area } \\
\text { error (\%) }\end{array}$ \\
\hline Ceramic ball & $16.6530^{a}$ & 16.3219 & 1.9882 & 31.5311 & 31.1472 & 1.2175 \\
Prolate ellipsoid & $9.3328^{b}$ & 9.3368 & 0.0428 & 23.0192 & 23.0508 & 0.1372 \\
Angled ellipsoid & $13.7176^{b}$ & 13.7456 & 0.2041 & 30.4192 & 30.5940 & 0.5746 \\
Oblong ellipsoid & $13.1888^{b}$ & 12.9976 & 1.4497 & 28.3884 & 28.0664 & 1.1342 \\
\hline
\end{tabular}

Plateau instability. The droplet along with its vapour wake accelerates towards the bulk liquid surface as shown in Fig.4a. As the evaporated nitrogen vapour diffuses away from the surface, the cold vapour could lead to the condensation of the water moisture in air to produce the observed wake structure shown in Fig.4a. Upon hitting the surface, the droplet deforms the surface while trying to penetrate it as seen in Fig.4b. While a smaller droplet just rebounds off the surface, a large droplet has sufficient momentum to break through the surface (Fig.4c). As the droplet breaks through, the large temperature difference between the bulk liquid and the $\mathrm{LN}_{2}$ droplet triggers a significant amount of heat transfer and hence a high evaporation rate, that eventually leads to a double interface configuration, i.e. a $\mathrm{LN}_{2}$ droplet trapped inside a bubble with the interface separating the bulk liquid and the gaseous nitrogen - as shown in Fig.4d. The droplet while in motion within the bubble continues to evaporate and feed vapour into the growing surrounding bubble (Fig.4e). The surface tension of both interfaces produces essentially smooth spherical/ellipsoidal shapes. The $\mathrm{LN}_{2}$ droplet, if large enough, will distort the bubble by a small amount due to gravity. This produces a visibly enhanced evaporation rate, and the droplet receives more thrust force from the evaporation process and becomes more mobile. As the evaporation proceeds, the bub- ble grows and rises to the surface while the droplet gets visibly smaller and obscured by the dense vapour now filled within the bubble. When the bubble grows large enough beyond the surface of the bulk liquid, the surface tension will no longer be able to stabilise the interface due to the drainage of the thin liquid interface under gravity as shown by Debregeas et al. [12]. As a result, the bubble collapses and releases its contents to the atmosphere as seen in Fig.4f. A lighter droplet would be released into the atmosphere upon surrounding vapour collapse but a heavier droplet will remain immersed in the bulk after bubble collapse as shown in Fig.4g and continue to evaporate within a new surrounding bubble (Fig.4h). A movie showing the typical repeated evaporation process of a nitrogen droplet can be found in the supplementary document A.

\subsection{Quantitative results from data analysis}

The radial growth of a nitrogen vapour bubble with different initial droplet sizes has been computed during the evaporation of $\mathrm{LN}_{2}$ droplets in 2-propanol (> 95\% purity), n-hexane (95\% purity), n-pentane (> 99\% purity) and methanol (99.8\% purity). These liquids were chosen firstly because of their extremely low miscibility in $\mathrm{LN}_{2}$, secondly, because their respective surface tensions are much lower than that of water $\left(72 \mathrm{mN} \mathrm{m}^{-1}\right)$. Besides, their densities being smaller than that of $\mathrm{LN}_{2}$, the released $\mathrm{LN}_{2}$ droplet could 


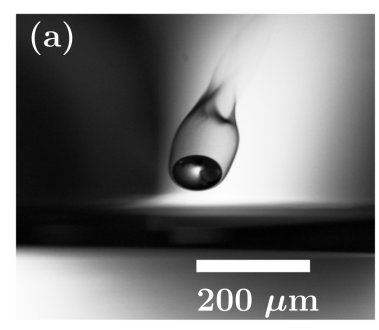

\section{(b)}
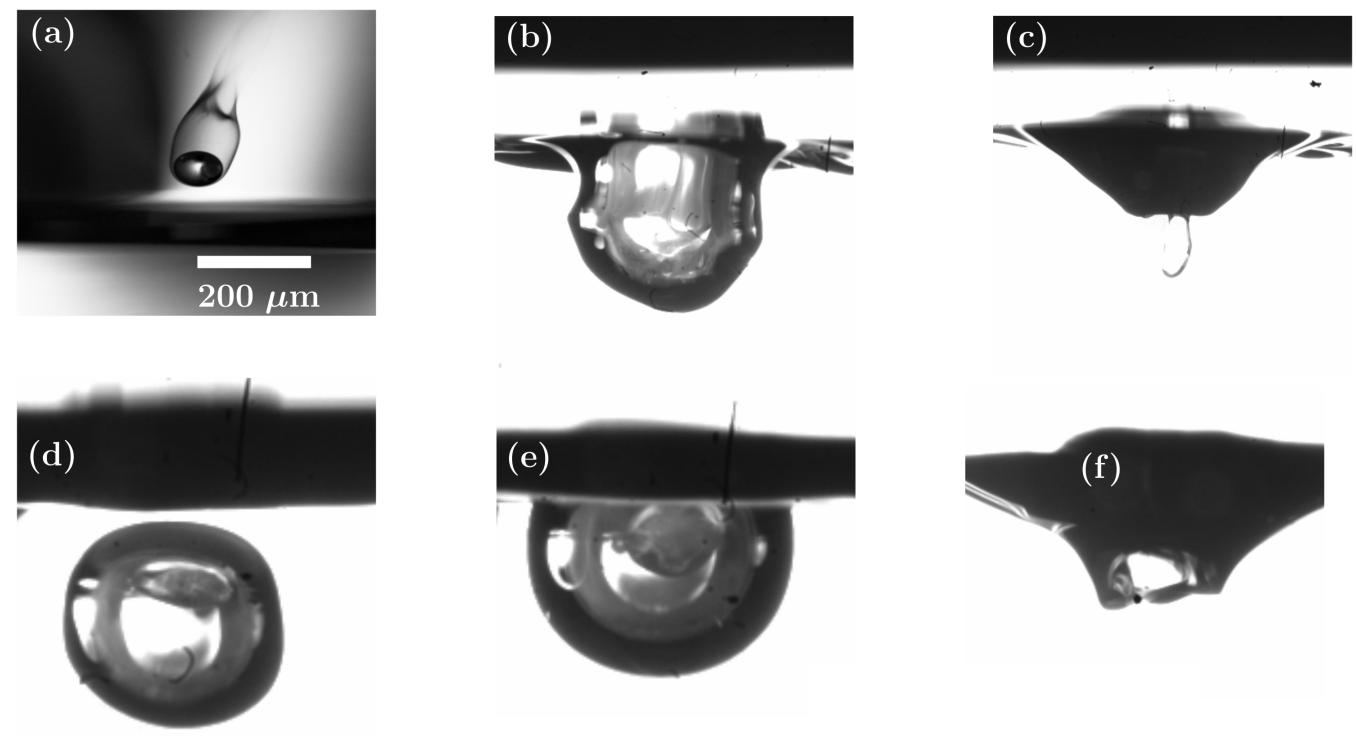

$(\mathrm{g})$

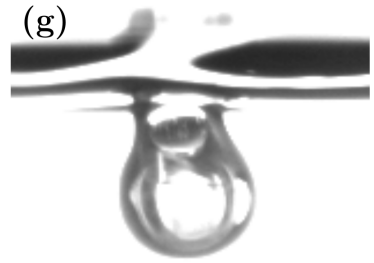

(h)

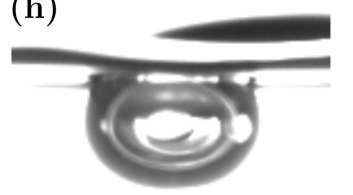

Figure 4: $\mathrm{LN}_{2}$ droplet immersion and boiling process in an immiscible bulk liquid. (a) before impact ; (b) impact/immersion ; (c) break-off ; (d) initial growth ; (e) growth (close to the surface) ; (f) bubble collapse ; (g) re-trapped droplet; (h) second bubble growth.

easily break through the surface.

A comparison of nitrogen droplets of varying volumes evaporating in 2-propanol, methanol, n-pentane and n-hexane is shown in Fig.5a-d. These results show that the bubble growth rate is noticeably affected by the initial droplet size consistently in all four tested fluids: larger droplets lead to more rapid growth. However, the initial droplet size is not the only parameter that affects the growth rate. Indeed, the latter can be slightly different when the initial droplet size is similar (see again Fig.5). This can be due to the slight difference in the droplet movement trajectory inside the bubble, as observed in the recorded videos, and the different fluid prop- erties, such as viscosity and surface tension (see §3.2.2 for a more detailed discussion).

\subsubsection{Scaling law for the bubble growth}

During the diffusion-controlled evaporation of a droplet in air at NTP, the decrease in the radius of a spherical droplet with time satisfies the classical $D^{2}$-law [13] of the form

$$
r_{d}^{2}=r_{0}^{2}-\alpha t,
$$

Where $r_{d}$ is the radius of the droplet at time $t, r_{0}$ is the initial radius of the droplet,and $\alpha$ is a diffusion parameter whose value depends on the condition of the surrounding vapour (e.g. whether Stefan flow is present or not, whether the bulk is confined or not). 

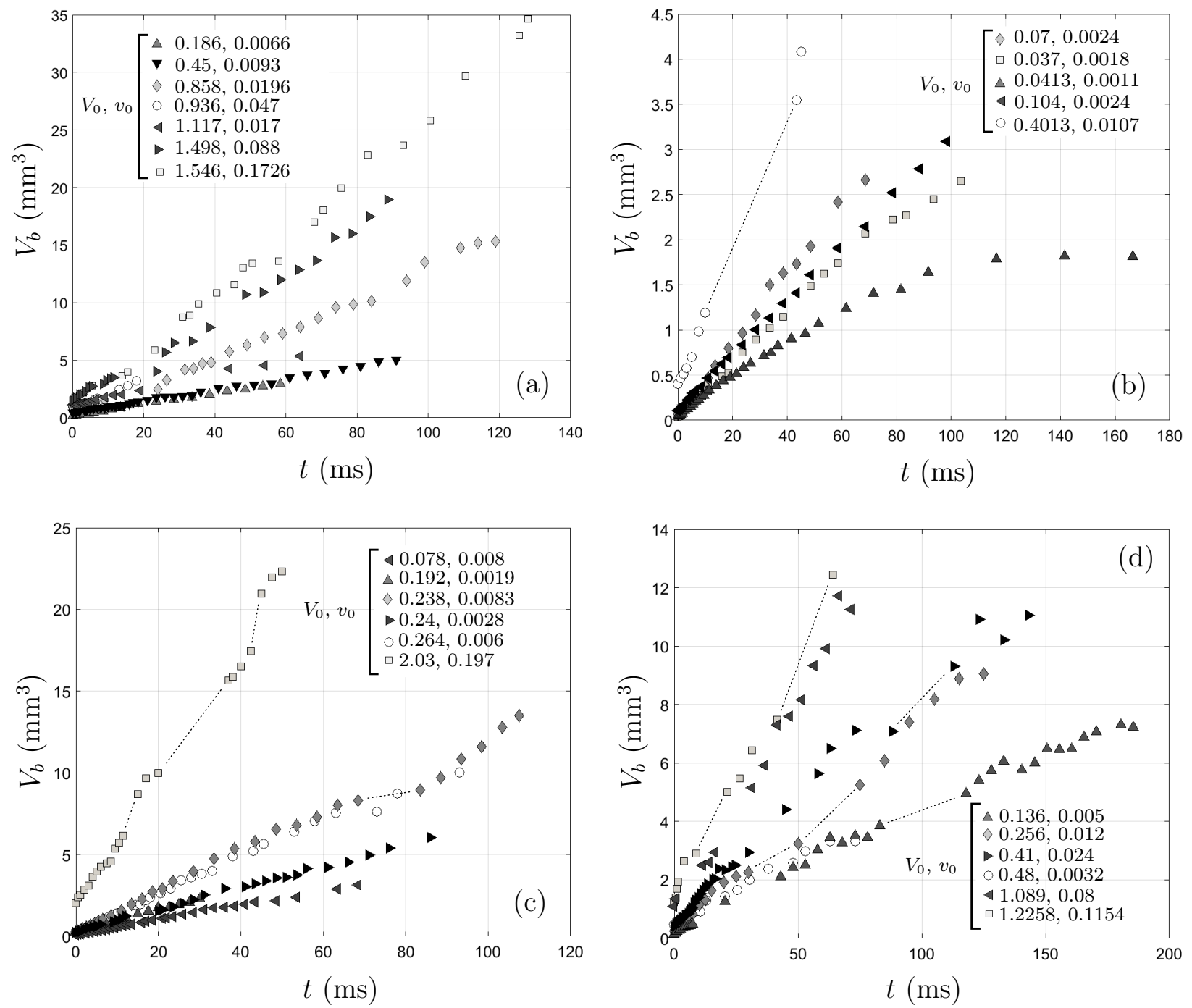

Figure 5: Bubble volume growth for a nitrogen droplet boiling in (a) 2-propanol, (b) methanol, (c) n-pentane and (d) n-hexane maintained at $294 \mathrm{~K}$. For each data set, the correspondence between the symbol and the initial bubble volume $V_{0}\left(\mathrm{~mm}^{3}\right)$ and the initial droplet volume $v_{0}\left(\mathrm{~mm}^{3}\right)$ is given in the legend. Gaps in the data, when measurement are not possible are filled with dotted lines to improve readability.

Please note that, mathematically, the $D^{2}$-law is developed based on the boundary condition that the effective latent heat (which takes into account the droplet heating) is a constant value and does not require the quasisteady-state assumption [14]. When the nitrogen droplet is in saturated condition, as in the experiments, the droplet heating is minimum hence the constant effective latent heat assumption and the the $D^{2}$-law is probably valid.

When the vapour phase thermal diffusivity is much larger than the liquid phase thermal diffusivity $(\approx$ two orders greater) and the vapour phase is at low pressures, then the vapour phase responds in a quasi-steady manner $[14,15,16]$. In such cases, the value of $\alpha$ can be computed from the mean properties of the vapour that surrounds the droplet such as its thermal conductivity $k_{b}$, specific 
heat $c_{p}$,the temperature difference between the droplet $\left(T_{d}\right)$ and its surrounding vapour $\left(T_{b}\right)$ as well as the droplet properties such as its density $\rho_{d}$ and its latent heat of vaporization $h_{f g}$ as shown below. $\alpha$ has the dimension of a diffusivity (i.e. $\mathrm{m}^{2} \mathrm{~s}^{-1}$ ).

$$
\alpha=\frac{2 k_{b}}{\rho_{d} c_{p}} \ln \left[1+\frac{c_{p}\left(T_{b}-T_{d}\right)}{h_{f g}}\right]
$$

Making the assumption that such a behaviour still holds in the case of a bubble confined droplet evaporation process and that the pressure inside the bubble is roughly equal to the atmospheric pressure $P_{a}$, one can show (see Appendix A) that the volume growth rate of the bubble at time zero can be written as

$$
v_{0}^{-1} \frac{d V_{b}}{d \tau}=\kappa, \text { where } \kappa=\frac{3}{2} \frac{\rho_{d} \mathcal{R} \bar{T}}{\mathcal{M}_{N_{2}} P_{a}}
$$

Where $V_{b}$ is the bubble volume at time $t, V_{0}$ is the initial volume of the bubble, $v_{0}$ is the initial volume of the droplet, $\rho_{d}$ is the density of nitrogen, $\mathcal{M}_{N_{2}}$ its molar mass, $\mathcal{R}$ is the ideal gas constant and $\tau=\alpha t / r_{0}^{2}$ is the dimensionless time based on the open space evaporation time $t_{e}=r_{0}^{2} / \alpha$. The definition of the mean temperature based on mass conservation considerations, is also given in appendix Appendix A, Eq.(A.5). Eq.(3) suggests that plotting $\left[V_{b}-V_{0}\right] / v_{0}$ as a function of $\tau$ is a relevant way to rescale the raw data presented in Fig.5. A graph of the bubble volume growth normalized by the initial droplet volume and plotted against the dimensionless time is shown in Fig.6. The plot scales most data well and confirms that the volume growth is proportional to time. Fig.6 shows that Eq.(3) with a correction factor of four (i.e. $\kappa^{\prime}=4 \kappa$ ) scales the data very well for the droplet evaporating in 2-propanol, methanol and $n$ - hexane with the deviation varying between $\pm 4-30 \%$. However, the data obtained

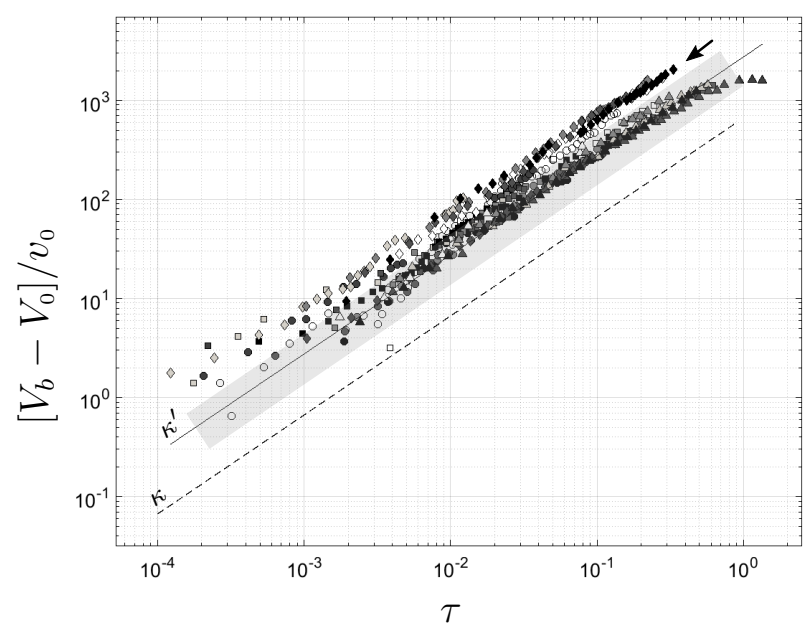

Figure 6: Experimental data rescaled using the relationship in Eq.(3). For 2-propanol, methanol, npentane and n-hexane. Except for n-pentane (black arrow), experimental points collapse fairly well on a single linear curve (see text for further explanations). Note that in this figure only, we used a single symbol for all data related to one specific fluid(circles/2propanol ; triangles/methanol ; diamonds/n-pentane ; squares/n-hexane). The shaded region represents the $30 \%$ deviation from the scaling correlation prediction.

when n-pentane was used as the bulk liquid show a larger growth rate (still proportional with time) with the deviation between $\pm 24-65 \%$. The effect of bulk liquid properties will be discussed in detail in the next section (§3.2.2).

An interesting observation is that although the droplet size rescales well using the diffusion controlled $D^{2}$-law, the growth rate of the bubble radius $R_{b}$ is proportional to the cube root of time $\left(R_{b} \sim t^{1 / 3}\right)$ for all the data. Although heat transfer was the dominant growth mechanism, the radial growth rate of the bubble differed from heat controlled growth regime $\left(R_{b} \sim t^{1 / 2}\right)$ of a vapour bubble growing in a superheated liquid [17]. Note that in one of the experiments, the bubble growth rate in methanol goes to zero as $\tau$ goes to 1 . It could be because the droplet 
within the bubble has been completely evaporated so the non-dimensional time is the appropriate characteristic time of the evaporation process. However, this could not be verified experimentally since the inside of the bubble was obscured by an opaque vapour cloud.

\subsubsection{Effect of bulk fluid properties}

The major bulk fluid properties that could potentially influence the droplet evaporation rates and hence the bubble growth rates are given in Table 2. The relative contribution of different forces and fluid properties to the growth rate of the bubble radius $R_{b}$ can be calculated from the Rayleigh-Plesset equation [18] together with the measured experimental data.

$$
\begin{gathered}
P_{b}-P_{l}=\underbrace{\rho_{l} R_{b} \frac{d^{2} R_{b}}{d t^{2}}+\frac{3}{2}\left(\frac{d R_{b}}{d t}\right)^{2}}_{\mathcal{P}_{i}} \\
+\underbrace{\frac{4 \nu_{l}}{R_{b}}\left(\frac{d R_{b}}{d t}\right)}_{\mathcal{P}_{v}}+\underbrace{\frac{2 \sigma_{l}}{R_{b}}}_{\mathcal{P}_{s}}
\end{gathered}
$$

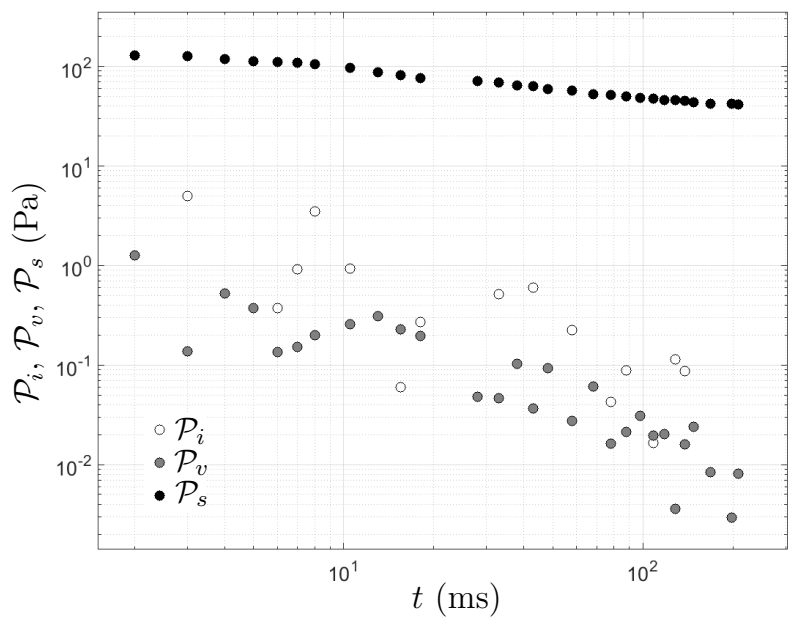

Figure 7: Respective amplitudes of the inertial, viscous and capillary terms in Eq.(4). Capillary terms are at least one order of magnitude larger than inertial and viscous terms. where pressure inside the bubble $P_{b}$ and bubble radius $R_{b}$ are functions of time. $P_{l}$ is the bulk liquid pressure $(\mathrm{Pa}), \rho_{l}$ is the bulk liquid density and $\nu_{l}$ is the kinematic viscosity of the bulk liquid $\left(\mathrm{m}^{2} \mathrm{~s}^{-1}\right)$. Note that the Rayleigh-Plesset equation assumes a uniform bubble pressure. The first two terms on the right hand side of Eq.(4) represent the inertial effects, the third term the viscous effects and the last term the surface tension effects. A typical result is shown in Fig.7 which shows that the surface tension effects are two orders of magnitude higher than the inertial effects and viscous effects. Therefore, the surface tension is the dominating term in the bubble growth and the simplified Young-Laplace equation shall be used to predict the bubble growth dynamics. If the same amount of nitrogen molecules are trapped inside the bubble and the surface tension is reduced, the bubble has to be larger so that the bubble pressure is reduced to a level which can be balanced by the reduced surface tension force. This explains why the bubbles in npentane tend to be the largest while bubbles in methanol tends to be smallest when compared to their initial droplet diameters.

In summary, section 3 showed that the bubble growth rate data can be scaled with a correction factor $\left(\kappa^{\prime}\right)$. The need for this correction could be due to the assumptions of a $\mathrm{LN}_{2}$ droplet evaporating in open air, the neglected effects of convection both within the bubble and in the bulk liquid and the use of a mean temperature $(\bar{T})$ to compute $\kappa$. In order to account for the droplet evaporation in a confined bubble with a non-uniform temperature profile within the bubble, a onedimensional analytical model was proposed as detailed in section (4.1). 
Table 2: Thermophysical properties of the bulk liquids at $294 \mathrm{~K}$.

\begin{tabular}{lcccc}
\hline & 2-Propanol & n-Hexane & n-Pentane & Methanol \\
\hline Density $\boldsymbol{\rho}_{\boldsymbol{l}}\left(\mathrm{kg} \mathrm{m}^{\mathbf{3}}\right)$ & 780.86 & 658.58 & 624.92 & 790.22 \\
Dynamic viscosity $\boldsymbol{\mu}_{\boldsymbol{l}}(\mathbf{P a ~ s})$ & $1.96 \times 10^{-3}$ & $0.3 \times 10^{-3}$ & $0.22 \times 10^{-3}$ & $0.577 \times 10^{-3}$ \\
Surface tension $\boldsymbol{\sigma}_{\boldsymbol{l}}\left(\mathbf{m} \mathbf{~ m}^{-\mathbf{1}}\right)$ & 21.74 & 18.50 & 16 & 22.95 \\
Thermal diffusivity $\boldsymbol{\alpha}_{\boldsymbol{l}}\left(\mathbf{m}^{\mathbf{2}} \mathbf{~ s}^{-\mathbf{1}}\right)$ & $70.0 \times 10^{-9}$ & $86.6 \times 10^{-9}$ & $78.7 \times 10^{-9}$ & $101.9 \times 10^{-9}$ \\
Solubility in $\mathbf{L N}_{\mathbf{2}}(\mathbf{m o l e}$ fraction$)$ & Not available & $7 \times 10^{-9}$ & $30 \times 10^{-9}$ & Not available \\
\hline
\end{tabular}

\section{Modelling and discussion}

\subsection{Conduction-based model}

A one-dimensional (1D) analytical model based on a simplified spherically symmetric geometry (see Fig.8), was developed to predict the bubble growth. The initial bubble size is taken as the measured bubble size at $t=0$. The key assumptions of the model are:

1. The $\mathrm{LN}_{2}$ droplet stays in the centre of the bubble and has a uniform temperature $T_{d}$ equal to the saturation temperature. Calculations show that the change of sensible heat of the droplet during evaporation is only a small fraction of the latent heat even for the largest droplets encountered in experiments so that the sensible heat is ignored in the energy analysis;

2. The temperature $T_{b}$ at the bubble boundary is equal to that of the bulk temperature (i.e. $T_{b}=T_{l}$ ); A separate steady state heat conduction analysis performed with the current bulk liquids that have high heat capacities and thermal diffusivities showed that this assumption is valid;

3. The bulk liquid is considered to be incompressible ;

4. Viscous and inertial forces in the bulk liquid can be ignored, as discussed in section 3.2.2, and the bubble is in quasiequilibrium condition so that the Young-
Laplace equation is applicable at different $R_{b}$;

5. The pressure within the bubble $P_{b}$ is considered as uniform, i.e., the pressure relaxation times are small and the nitrogen vapour follows the ideal gas law (compressibility factor of nitrogen at 1 bar saturation condition is 0.96 [19] so it is very close to idea gas behaviour);

6. Nitrogen and the bulk liquids are immiscible so mass transfer across the bubble boundary is negligible;

7. The convective heat transfer in both the bulk fluids and the nitrogen vapour inside the bubble are ignored .

8. It is further assumed that a quasi-steady temperature profile inside the nitrogen vapour is established instantaneously at each time step and can be calculated based on the steady-state heat conduction analysis.

The quasi-steady assumption may not be valid due to (a) finite thermal diffusivity of the nitrogen vapour, (b) radial convection due to the expansion of the bubble, (c) the recirculation convection within the bubble due to the motion of the droplet. To evaluate the effect of thermal diffusivity and radial convection, we calculate the lower bound of the Peclet number $(\mathrm{Pe})$ from the initial values of the bubble radius $R_{0}$ and the initial bubble radial growth rate $d R_{b} /\left.d t\right|_{t=0}$ (considered as a typical velocity), which lies in the range 

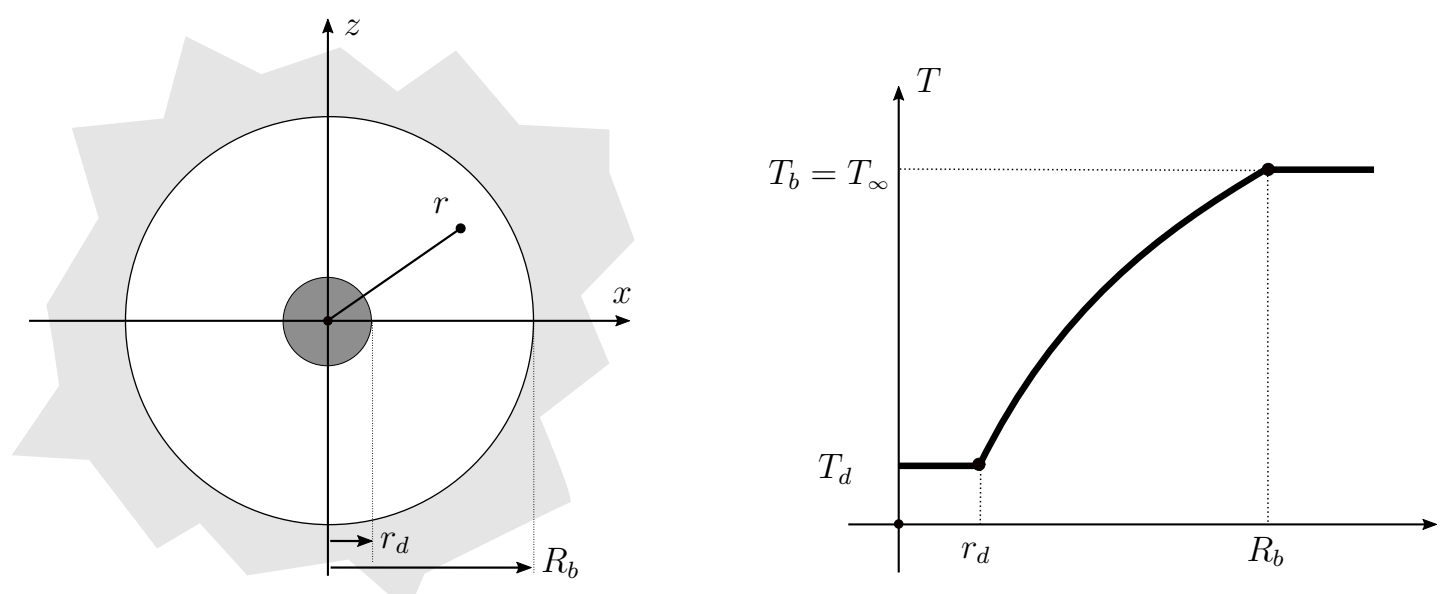

Figure 8: Geometrical configuration used for the model (spherical symmetry).

$[0.2-1.1]$, so the quasi-steady state assumption is not fully justified in conditions when $\mathrm{Pe} \geq O(1)$. The experimental data shows that the droplet is very mobile during its evaporation so the effect of recirculation convection could be significant. Considering the full modelling will require a very demanding detailed three-dimensional transient model, as a first step to model the complex evaporation process, we will ignore these effects and aim to quantify the error introduced by the quasi-steady state assumptions and find a way to correct it if necessary.

The steady-state one dimensional heat conduction in radial direction is given by

$$
\nabla^{2} T=0
$$

Integrating the above equation between the droplet and bubble radii, and applying the boundary conditions: $T_{b}=T_{l}$ at $r=R_{b}$, $T=T_{d}$ at $r=r_{d}$, leads to the following temperature profile:

$$
T(r)=-\frac{\left(T_{l}-T_{d}\right) R_{b}}{R_{b}-r_{d}} \frac{r_{d}}{r}+\frac{T_{l} R_{b}-T_{d} R_{d}}{R_{b}-r_{d}}
$$

The heat flux $q$ transferred into the droplet from the surrounding nitrogen can then be calculated from Eq.(6) as

$$
\left.q=k_{b} \frac{d T}{d r}\right\rfloor_{r=r_{d}}=k_{b} \frac{T_{l}-T_{d}}{R_{b}-r_{d}} \frac{R_{b}}{r_{d}}
$$

Based on Assumption 1, the mass of $\mathrm{LN}_{2}$ evaporated during a time $d t$ (assuming the temperature profile remains unchanged) can be calculated from:

$$
d m_{d}=\frac{4 \pi r_{d}^{2} q}{h_{f g}} d t
$$

The previous equation can be rewritten in terms of molar volume

$$
d n=\frac{4 \pi r_{d}^{2} q}{h_{f g} \mathcal{M}_{N_{2}}} d t
$$

and the change in the droplet radius due to evaporation is given by

$$
d r_{d}=-\frac{\mathcal{M}_{N_{2}}}{\rho_{b} 4 \pi r_{d}^{2}} d n
$$

The evaporated nitrogen vapour will then add up to the existing vapour which leads to an increase in the bubble pressure. Hence the bubble will grow to re-establish the force balance at the boundary. Based on the Assumptions 4 and 5 , the nitrogen vapour density 
profile inside the bubble can be represented as

$$
\rho_{b}(r)=\frac{P_{b} \mathcal{M}_{N_{2}}}{\mathcal{R} T(r)} \text { with } P_{b}=P_{l}+\frac{2 \sigma_{l}}{R_{b}}
$$

where $P_{l}=10^{5} \mathrm{~Pa}$ as the bulk liquid is open to atmosphere and the additional pressure imposed on the bubble due to the liquid head $\left(\rho_{l} g R_{b}\right)$ is small and hence neglected.

The total number of moles in the vapour layer can then be represented as:

$$
n=\frac{4 \pi}{\mathcal{R}}\left(P_{l}+\frac{2 \sigma_{l}}{R_{b}}\right) \int_{r_{d}}^{R_{b}} \frac{r^{2}}{T(r)} d r
$$

The change in the bubble size $d R_{b}$ when $d n$ extra moles of nitrogen are added to the vapour layer can then be obtained by differentiating Eq.(12), which yields

$$
\begin{gathered}
d R_{b}=\left[\frac{\mathcal{R}}{4 \pi} d n+\left(P_{l}+\frac{2 \sigma_{l}}{R_{b}}\right) \frac{r_{d}^{2}}{T_{d}} d r_{d}\right] / \\
{\left[\left(P_{l}+\frac{2 \sigma_{l}}{R_{b}}\right) \frac{R_{b}^{2}}{T_{b}}-\frac{2 \sigma_{l}}{R_{b}^{2}} \int_{r_{d}}^{R_{b}} \frac{r^{2}}{T(r)} d r\right]}
\end{gathered}
$$

The droplet and bubble radii can be updated using a first order incrementation i.e. $R_{b}(t+\delta t)=R_{b}(t)+d R_{b}, r_{d}(t+\delta t)=$ $r_{d}(t)+d r_{d}$. These updated values can then be used to perform the steady-state heat conduction analysis at the next time and calculate the amount of evaporated nitrogen, the change in radii $d R_{b}$ and $d r_{d}$ and so on. In order to choose the appropriate time step that produced a largely unchanged temperature profile in the vicinity of the droplet, different values of $d t$ have been tested and the value $d t=0.5 \mathrm{~ms}$ was retained.

\subsection{Confrontation to experimental data}

The bubble growth rate predicted by the model with pure conductive limit $\left(k_{b}\right)$ for the smallest droplet evaporating in 2-propanol is shown in Fig.9a,together with experimental data. Only one data set is shown to improve legibility. Fig.9 a shows that the model captures the trends fairly well for different bulk fluids and initial droplet sizes but consistently underestimates the growth rate.

This is firstly due to an oversimplification of the fixed droplet position - i.e. assumption 1. Indeed, the droplet was observed to move around within the bubble and spends some of its life close to the bubble lower boundary as sketched in Fig.10. Secondly, the convection effects both within the bubble and the bulk liquid are not captured by the model with pure conduction.

In order to estimate the maximum evaporation rate while taking into account the droplet positioned close to the bubble boundary, the model detailed in section 4.1 was modified to account for the effects shown in Fig.10. An expression for predicting the vapour layer thickness $\delta$ of a small spherical evaporating droplet on a solid was given by Biance et al. [20] in the following form:

$$
\delta=\left[\frac{3 k_{b}\left(T_{b}-T_{d}\right) \mu_{b}}{4 h_{f g} \rho_{b} \sigma_{d} a^{2}}\right]^{1 / 4} r_{d}^{5 / 4}
$$

where $a$ is the capillary length. The previous expression was derived by equating the evaporating mass flow due to heat transfer into the droplet and the mass flow due to the pressure exerted on the film by the droplet lubrication theory. The capillary length of the largest droplet found in the experiments is larger than the radius of the droplet $(a=$ $\left.\left[\sigma_{d} /\left(\rho_{d} g\right)\right]^{1 / 2} \simeq 1.18 \mathrm{~mm}>r_{d}=0.36 \mathrm{~mm}\right)$, indicating that the droplet is nearly spherical. Considering the bulk liquid as an incompressible smooth surface, Eq.(14) is likely to be a good approximation for predicting the vapour layer thickness between the droplet and bubble-bulk liquid interface. The evaporation rate of the droplet was then com- 

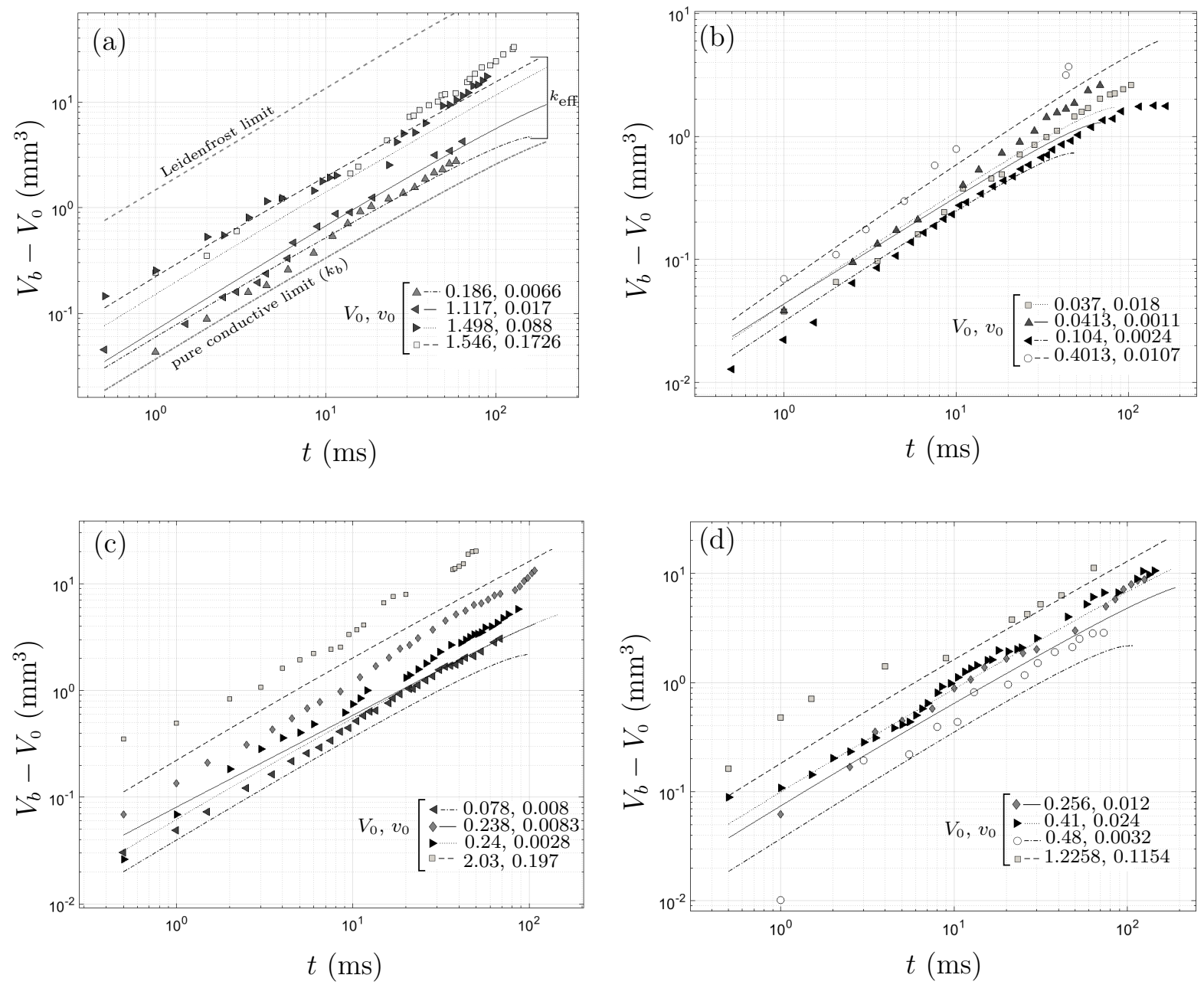

Figure 9: Predicted bubble growth rates with an effective thermal conductivity $\left(k_{\text {eff }}=1.6 \times k_{b}\right)$ against experimental data for a nitrogen droplet boiling in (a) 2-propanol, (b) methanol, (c) n-pentane and (d) n-hexane maintained at $294 \mathrm{~K}$. For each data set, the correspondence between the symbol and the initial bubble volume $V_{0}\left(\mathrm{~mm}^{3}\right)$ and droplet volume $v_{0}\left(\mathrm{~mm}^{3}\right)$ is given in the legend. Note that, for each liquid, two or three data sets have been removed to improve legibility.

puted by considering quasi-steady heat transfer across the vapour layer, with the heat acting on the lower hemisphere surface area of the droplet. The bubble growth rate was then computed as in the earlier case. The modelling results for the largest droplet evaporating in 2-propanol is also shown in Fig.9a (Leidenfrost limit). Only one data set is shown to improve legibility. The results show that the model accounting for evaporation under Leidenfrost condition overestimates the bubble growth rates for all the initial conditions considered.

To correct for the simplified eccentric con- figuration, an empirical effective thermal conductivity $k_{\text {eff }}=1.6 \times k_{b}$ was introduced to account for the motion of the droplets and the effects of convection within the bubble. The modelling results using $k_{\text {eff }}$ were plotted in Fig.9 for different initial droplet sizes and bulk liquids.

The corrected predications are in good agreement with data obtained for different droplet radii while evaporating in 2-propanol, methanol and n-hexane. For n-pentane, the predicted growth rates were still lower than the experimental data. The effective thermal conductivity produced a good fit for the 


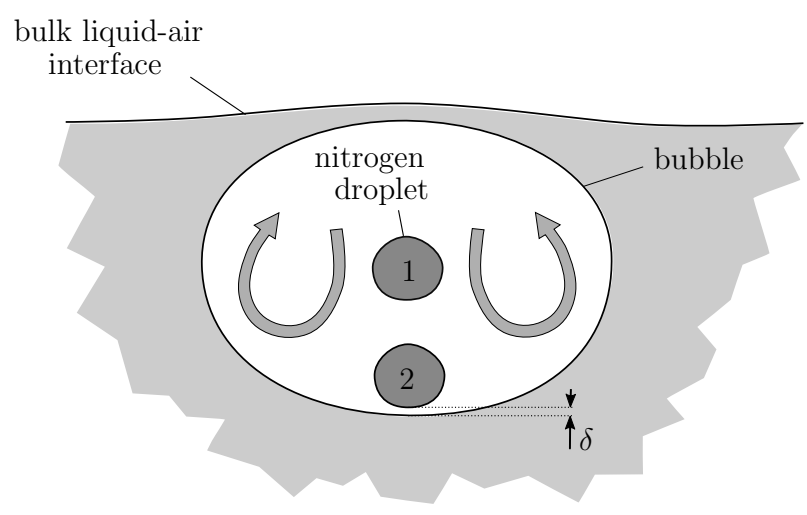

Figure 10: Breaking of spherical symmetry and convection (due to density gradients and bubble shape fluctuations) are taken into account by introducing an effective thermal conductivity $k_{\text {eff }}$ - see text for further comments.

experimental data. A more insightful understanding and accurate calculation of the $k_{\text {eff }}$ in different conditions will likely need a much more complex multi-dimensional model that takes into account the detailed motions of the droplet and the relevant convective current inside the bubble.

\section{Heat transfer rates}

The heat fluxes to the bubble surface were computed from the bubble growth obtained from experimental data using the following expression

$$
q_{b}=\frac{\rho_{b} h_{f g}}{A_{b}} \frac{d V_{b}}{d t}
$$

where $A_{b}$ is the estimated surface area of the bubble. The assumption made to obtain the previous expression is that the heat transferred into the bubble is used to evaporate the $\mathrm{LN}_{2}$ into nitrogen vapour and then heat it to the arithmetic mean temperature between the bulk and saturation temperature. Fig.11 shows the experimental heat fluxes to the bubble along with the results produced by the model. The decreasing trend of heat flux with time is well captured by the model; however, the experimental data is scattered due to the evaporation process being influenced by the motion of the droplet. The heat fluxes are higher at the start, gradually reducing with time as the droplet reduces in size and the bubble grows in size.

Heat fluxes to the droplet surface were computed from Eq.(16) assuming the droplet surface area $\left(A_{d}\right)$ remains fairly constant during the evaporation process. This assumption seems valid since the droplet volume does not vary significantly (typically $\Delta v_{d} / v_{d} \sim 1 \%$ when $\Delta t=5 \mathrm{~ms}$ ) as observed during experiments.

$$
q_{d}=\frac{\rho_{b} h_{f g}}{A_{d}} \frac{d V_{b}}{d t}
$$

The droplet evaporating in n-pentane has the highest heat flux while the droplet evaporating in methanol has the lowest heat flux. A comparison between the heat fluxes obtained from experiments was done with pool boiling data available for $\mathrm{LN}_{2}$. The average value of heat flux obtained in current experiments was $25 \mathrm{~W} \mathrm{~cm}^{-2}$, which is higher than the heat flux (based on the heating surface area) measured during pool boiling of liquid nitrogen $(6.5 \mathrm{~W}$ $\mathrm{cm}^{-2}$ ) for the same temperature difference [21]. Considering the cumulative droplet surface area in a cryogenic spray is much larger than the area of the heating surface in typical pool boiling chamber, the direct injection of cryogenic sprays into a warmer immiscible liquids can provide a much more efficient way to evaporate the cryogen.

\section{Conclusions}

An experimental technique for precisely measuring the droplet and bubble sizes during evaporation of a liquid nitrogen droplet 

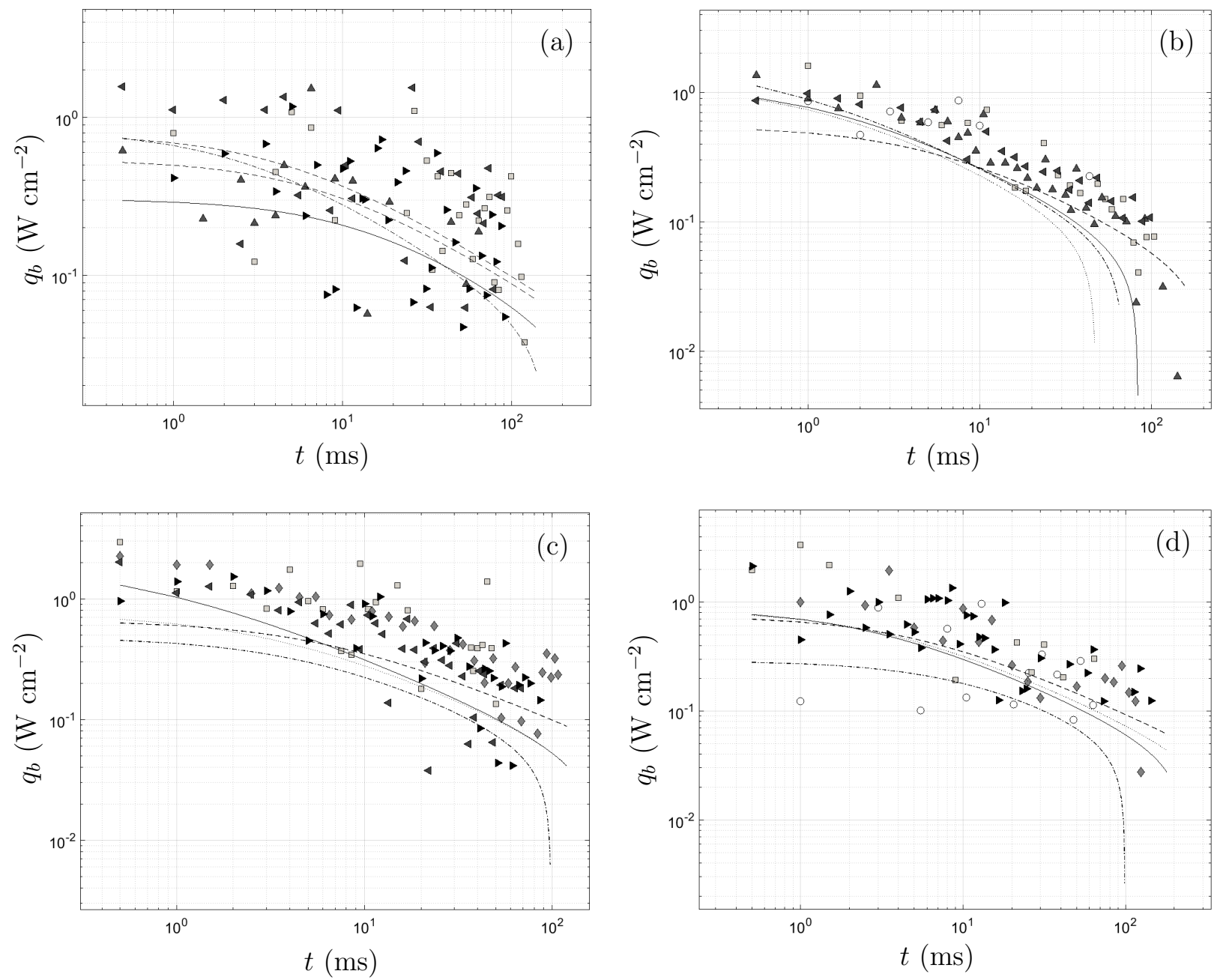

Figure 11: Heat fluxes ( $\mathrm{W} \mathrm{cm}^{-2}$ ) into a nitrogen bubble growing in (a) 2-propanol, (b) methanol, (c) npentane and (d) n-hexane maintained at $294 \mathrm{~K}$ together with the modelling results. For the correspondence between symbols and lines, see legends in Fig.9.

in different immiscible liquids has been presented. Based on authors' best knowledge, this is the first study to visualise and quantify the detailed evaporation process of an isolated cryogenic liquid droplet immersed into a high temperature immiscible bulk liquid in the 'film boiling' regime. The experimental results showed that the initial droplet size has a dominant influence on the surrounding bubble growth while other bulk liquid properties also influenced the surrounding bubble growth. The scaling analysis based on the $D^{2}$-law for droplet evaporation suggested the following scaling law

$$
\frac{V_{b}-V_{0}}{v_{0}}=\kappa^{\prime}\left(\frac{\alpha t}{r_{0}^{2}}\right)
$$

where $\kappa^{\prime}$ is the empirical scaling coefficient based on experimental data. This scaling correlation captures the bubble growth process reasonably well. A one dimensional quasisteady state heat conduction model with an 
effective thermal conductivity has been presented that successfully captures the experimental bubble growth rates with different initial droplet sizes and bulk liquids. The average heat flux $\left(25 \mathrm{~W} \mathrm{~cm}^{-2}\right)$ based on the nitrogen droplet surface area was significantly higher than that of the pool boiling of liquid nitrogen which is based on the heating surface area.

In summary, the results obtained through this study confirms that a spray of cryogenic droplets boiling in an immiscible liquid can provide an efficient method for utilizing the stored exergy in cryogenic liquids for cooling and energy applications, especially when the bulk liquid has a low surface tension. The developed model provides a convenient way to predict the evaporation rate of a single cryogenic droplet immersed into an immiscible liquid, which can be used together to predict the evaporation rates of a cryogenic sprays in the future.

\section{Acknowledgments}

Part of the work presented in this paper has received funding from the Dearman Engine Company, UK. The financial support is gratefully acknowledged by the authors'.

\section{Conflict of interest}

The authors declare that there is no conflict of interest.

\section{Appendix A. Rescaled variables}

The objective of this appendix is to justify the choice made in the rescaling of the raw data. In the case of an unconfined droplet whose evaporation is dominated by diffusion processes (mass and heat transfers), the time evolution of the droplet radius is properly rendered by a $D^{2}$-law $[13,22]$. The decrease in time of the radius $r_{d}$ of a drop of initial radius $r_{0}$ is then given by a generic equation of the form

$$
r_{d}^{2}=r_{0}^{2}-\alpha t
$$

where the coefficient $\alpha$ has the dimension of a diffusivity. Differentiating Eq.(A.1) with respect to time and mutiplying by $2 \pi r_{d}$ leads to

$$
\frac{d v_{d}}{d t}=-2 \pi \alpha\left(r_{0}^{2}-\alpha t\right)^{1 / 2}
$$

which governs the decrease of the droplet volume in time. By introducing in the previous equation the density of nitrogen $\rho_{d}$ and its molar weight $\mathcal{M}_{N_{2}}$, one obtains the number of moles of nitrogen $n$ leaving the drop per unit time due to evaporation:

$$
\frac{d n}{d t}=-\frac{2 \pi \alpha \rho_{d}}{\mathcal{M}_{N_{2}}}\left[r_{0}^{2}-\alpha t\right]^{1 / 2}
$$

In the following, we assume that (i) the pressure in the bubble is constant in time and close to the atmospheric pressure $P_{a}$, (ii) nitrogen can be considered as an ideal gas, (iii) the change in volume due to the shrinking of the droplet can be neglected. We also make the rough additional assumptions that (iv) the bubble cavity is at mean temperature $\bar{T}$ constant in time. The previous equation can thus be rewritten in terms of volume change as

$$
\frac{d V_{b}}{d t}=\frac{2 \pi \alpha \rho_{d} \mathcal{R} \bar{T}}{\mathcal{M}_{N_{2}} P_{a}}\left[r_{0}^{2}-\alpha t\right]^{1 / 2}
$$

where $V_{b}$ is the volume of the bubble. For conservation reasons (see $\S 4.1$ ), the mean temperature $\bar{T}$ used in Eq.(A.4) is the space average temperature defined by

$$
\bar{T}=\left[\frac{4 \pi}{V_{0}-v_{0}} \int_{r_{0}}^{R_{0}} \frac{r^{2}}{T(r)} d r\right]^{-1}
$$

where the temperature profile is the steady temperature profile given by Eq.(6) at time 
zero. In the small times limit $t \ll t_{e}$, Eq.

(A.4) finally simplifies to

$$
v_{0}^{-1} \frac{d V_{b}}{d \tau}=\kappa, \text { where } \kappa=\frac{3}{2} \frac{\rho_{d} \mathcal{R} \bar{T}}{\mathcal{M}_{N_{2}} P_{a}}
$$

and $\tau=t / t_{e}$. 
unsrt

\section{References}

[1] IPCC report. Climate Change 2014: Synthesis Report. Contribution of Working Groups I, II and III to the Fifth Assessment Report of the Intergovernmental Panel on Climate Change ipcc, Geneva, Switzerland. page 151 pp., 2014.

[2] Ordonnez C. Liquid nitrogen fueled, closed Brayton cycle cryogenic heat engine. Energy Convers. Manag., 41:331-341, 2000.

[3] N. Owen, H. Clarke, D. Charters, J. Trembley, C. Garner, S. Mohr, A. Williams, and H. Zhao. Development of a high efficiency liquid-air engine for cooling, heat recovery and power. proceedings of the Vehicle Thermal Management Systems Conference (VTMS 12), 6, 2015.

[4] H. Clarke. A novel cryogenic energy system for zero emission vehicles. PhD thesis, Queen Mary University of London, UK, 2011.

[5] S.O. Awonorin. Evaporation rates of freely falling liquid nitrogen droplets in air. Heat Transfer Engineering, 10:1:26-36, 1989.

[6] D. Wen, H. Chen, Y. Ding, and P. Dearman. Liquid nitrogen injection into water: Pressure build-up and heat transfer. Cryogenics, 46:740-748, 2006.

[7] H. Clarke, A. Martinez-Herasme, R. Crookes, and D. S. Wen. Experimental study of jet structure and pressurisation upon liquid nitrogen injection into water. Int. J. Multiph. Flow, 36:940-949, 2010.

[8] V. Nakoryakov, I. Mezentsev, A. Meleshkin, and D. Elistratov. Visualization of physical processes occurring on liquid nitrogen injection into water. J. Eng. Thermophys., 24:322-329, 2015 .

[9] R. Clift, J. Grace, and M. Webber. Bubbles, drops, and particles. Dover publications Inc., 2005 .

[10] W. Burger and M. Burge. Digital image processing an algorithmic introduction using java. Springer, 2008.

[11] R. McLaughlin. Randomized Hough Transform: Improved ellipse detection with comparison. Pattern Recognit. Lett., 19:299-305, 1998.

[12] G. Debrégeas, P.-G. de Gennes, and F. Brochard-Wyart. The Life and Death of "Bare Viscous Bubbles. Science, pages 1704-1707, 1998.

[13] D. Spalding. Combustion and mass transfer: a textbook with multiple-choice exercises for engineering students. Pergamon Press, 1979.

[14] W. Sirignano. Fluid dynamics and transport of droplets and sprays. Cambridge university press, 2010. 
[15] F. A. Williams. On the assumptions underlying droplet vaporization and combustion theories. The Journal of Chemical Physics, 33(1):133-144, 1960.

[16] J. Bellan and M. Summerfield. Quasi-steady gas phase assumption for a burning droplet. AIAA Journal, 14(7):973-975, 1976.

[17] V. Carey. Liquid vapor phase change phenomena: An introduction to the thermophysics of vaporization and condensation processes in heat transfer equipment. Second Edition, CRC Press, 2018.

[18] M. S. Plesset and S. A. Zwick. The Growth of Vapor Bubbles in Superheated Liquids. J. App. Phys., 25(4):493-500, 1954.

[19] R. Span, E. W. Lemmon, R. T. Jacobsen, W. Wolfgang, and A. Yokozeki. A reference equation of state for the thermodynamic properties of nitrogen for temperatures from 63.151 to 1000 $\mathrm{k}$ and pressures to 2200 mpa. Journal of Physical and Chemical Reference Data, 29(6):13611433, 2000.

[20] A. Biance, C. Clanet, and D. Quéré. Leidenfrost drops. Phys. Fluids, 15:1632-1637, 2003.

[21] M. Kida, Y. Kikuchi, O. Takahashi, and I. Michiyoshi. Pool-boiling heat transfer in liquid nitrogen. J. Nucl. Sci. Technol., 18:501-513, 1981.

[22] I. Langmuir. The evaporation of small spheres. Phys. Rev., 12:368-370, 1918.

IJHMT2019 